\title{
Topographic and Tomographic Indices for Detecting Keratoconus and Subclinical Keratoconus: A Systematic Review
}

\section{David Smadja}

\section{ABSTRACT}

Purpose: To provide an overview of the topographic and tomographic indices developed for detecting keratoconus (KC) and subclinical keratoconus.

Methods: Literature review of studies describing and testing KC indices as well as indices developed for improving the sensitivity of subclinical KC detection.

Results: Several indices, based on anterior and posterior curvature measurements, corneal spatial distribution or posterior corneal elevation have been developed for improving the detection of KC and subclinical KC. However, to date, none of them could reach, alone, sufficient discriminating power for differentiating the mildest forms of the disease from normal corneas. New detection programs, based on a combination of corneal indices, and generated using artificial intelligence emerged recently and helped to significantly improve the subclinical KC detection.

Conclusion: The combination of topographic and tomographic corneal indices has helped to significantly improve the sensitivity of subclinical KC detection. However, combining these morphological indices to wavefront and biomechanical analyses of the cornea will certainly further improve the sensitivity of the future screening tests.

Keywords: Keratoconus, Topographic, Tomografic, Form fruste keratoconus, Scheimpflug, Placido, Screening.

How to cite this article: Smadja D. Topographic and Tomographic Indices for Detecting Keratoconus and Subclinical Keratoconus: A Systematic Review. Int J Kerat Ect Cor Dis 2013;2(2):60-64.

\section{Source of support: Nil}

Conflict of interest: None declared

\section{INTRODUCTION}

Identifying corneas with risk of developing iatrogenic ectasia after laser in situ keratomileusis (LASIK) remains the major concern of the preoperative refractive surgery screening. Over the past 20 years, significant technological advancements have been achieved in the field of anterior segment imaging for improving keratoconus detection. Modern corneal imaging technologies are able to provide far more details on corneal structure, thereby, greatly enhancing our ability to investigate corneal properties.

This article aims to provide an overview of the topographic and tomographic indices that have been developed with the various imaging technologies for improving the sensitivity of keratoconus and subclinical $\mathrm{KC}$ detection.

\section{Indices based on Placido Topography}

Corneal topography has been introduced in the mid 1980s and remains until now the standard of care for preoperative patient screening before keratorefractive surgery. Its principle is based on the computerized analysis of corneal images obtained from the reflection of Placido disk onto the corneal surface. Corneal topography has represented a true revolution in the diagnosis of ectatic disorder and has been found sensitive for the detection of early keratoconus, prior to loss of corrected visual acuity and biomicroscopic findings. ${ }^{1}$ The emerging interest in laser keratorefractive surgery along with the onset of post-LASIK ectasia cases associated with early keratoconus, ${ }^{2}$ have led to the development of several literature-validated keratoconus detection schemes based on corneal topography. ${ }^{3,4}$ Among these multiples keratoconus detection indexes, three of them have been widely used and implemented in most of the corneal topographers.

In 1989, the seminal works of Rabinowitz et al. ${ }^{5}$ have helped differentiating between normal and suspect keratoconus by developing the 'I-S ratio', which represents the amount of steepening of the inferior cornea compared with that of the superior cornea. The authors have considered a 1.4-1.9 D value as consistent with keratoconus suspect while greater value would be consistent with clinical keratoconus. In subsequent works, they refined their finding and found a cut-off value of $0.8 \mathrm{D}$ for distinguishing between normal eye and suspect keratoconus. ${ }^{6}$ Later, Maeda and Klyce have developed the keratoconus prediction index (KPI) to further help in differentiating keratoconus from other corneal irregularities patterns. ${ }^{7}$ This index is derived from eight other videokeratography indices, including the differential sector index (DSI), the opposite sector index (OSI), the center/surround index (CSI), the surface asymmetry index (SAI), the irregular astigmatism index (IAI), and the percent analyzed area (AA). The third widely spread indice is the KISA\% index proposed by Rabinowitz in $1999 .{ }^{6}$ The KISA\% index is calculated from a combination of four videokeratography parameters: the central keratometry power, the I-S value, the corneal simulated astigmatism (SimCyl) and the SRAX index, which is the representation of the irregular astigmatism (smallest angle between 2 steep radii subtracted from $180^{\circ}$ ). In his original study aiming to test this new algorithm, Rabinowitz et al. have demonstrated that KISA\% index set at 100 was 
highly sensitive and specific for diagnosing keratoconus, with $99.6 \%$ of keratoconus patients correctly classified. However, the correct classification rate on the same sample for the other indices tested were $88.6 \%$ with the KPI, and $95.7 \%$ with the I-S ratio. In addition, the authors have suggested that a KISA\% ranging from 60 to $100 \%$ should raise the diagnosis of suspect keratoconus.

More recently, Abad and Belin have proposed a novel topographic curvature pattern, called the 'vertical D' after its retrospective detection in patients who developed postLASIK ectasia. ${ }^{8}$ The vertical D reflects the horizontal asymmetry and is described as a topographic pattern with nasal-temporal asymmetry comprising an orthogonal bow tie with the tips connecting on the temporal side and leaving a small low-power island connected with the center point of the map. In their subsequent prospective crosssectional study on 1168 consecutive potential refractive surgical candidates, the authors have found the presence of the vertical $\mathrm{D}$ in $0.34 \%$ of eyes and all were associated in addition, to parameters evoking suspect keratoconus, such as central pachymetry thinner than $500 \mu \mathrm{m}$ and/or posterior corneal protrusion greater than $20 \mu \mathrm{m}$.

The cone location and magnitude index (CLMI) is one of the latest development in the field of keratoconus detection based on corneal topography. ${ }^{9}$ This index aims to detect keratoconic patterns and quantify cone location and curvature magnitude with the concern of being able to track the progression of the disease through the curvature magnitude of the cone. In addition, this index has the advantage of being independent of a specific platform and thus applicable with different corneal topography system. A complete separation of normals and keratoconus eyes was achieved with $100 \%$ specificity and $100 \%$ sensitivity when using a validation set.

\section{Placido Topography vs Corneal Tomography}

Although, corneal topography has been found sensitive for detecting keratoconus prior to clinical biomicroscopic findings, it has been shown clear evidences of subclinical keratoconus in corneas undergoing tomographic analysis while they were considered normal by the various topographic keratoconus detection indices. ${ }^{10,11}$ This finding further feeds the debate on the location of the first detectable sign of subclinical keratoconus, whether it is subtle changes in anterior surface curvature seen with Placido disk or posterior surface changes detected only by tomography.

Several recent studies have pointed out the significant role of corneal epithelium in reducing and smoothing corneal topographic irregularity ${ }^{12}$ as well as in masking the presence of an underlying cone on the anterior surface in early keratoconus. ${ }^{13,14}$ This ability that has the epithelium to remodel itself to compensate for stromal surface abnormalities and thus to affect the topographic aspect of the cornea has been put forward for explaining early detection of keratoconus at a posterior surface level. ${ }^{15}$ Indeed, corneal measurement with Placido disk technology is exclusively limited to the anterior surface analysis. However, corneal tomography allows for a more complete analysis of the corneal architectural properties, such as elevation-derived parameters, posterior surface analysis or pachymetric spatial profile. Tomographic imaging includes different technologies, such as horizontal slit scanning, rotational Scheimpflug imaging, arc scanning with very high-frequency ultrasound, and optical coherence tomography, and is available in several commercial instruments.

The occurrence of ectasia cases after uneventful LASIK with no pre-existing recognized risk factors detected by traditional screening criteria such as refraction, age and residual stromal bed, along with Placido disk topography and single-point thickness measurement, has further demonstrated the need for these newer imaging technologies in order to improve the sensitivity and specificity of the current screening tests. ${ }^{16}$

\section{Indices based on Scheimpflug Imaging and Slit- Scanning Technology}

\section{Posterior Surface Modifications}

Modifications of the posterior surface in keratoconus have been well described with various imaging systems, and include an increase in curvature, astigmatism, and asymmetry when compared to normal corneas, as well as a forward bulging in elevation maps. ${ }^{15,17,18}$

In early forms of keratoconus, significant differences in posterior surface features when compared to normal corneas have also been reported with, however, a lower discriminant power and overlaps of values between these two populations. Schlegel et al have reported significant greater posterior astigmatism, posterior elevation and a more prolate posterior surface in suspect keratoconic eyes compared to normal eyes with the Orbscan IIz system (Bausch \& Lomb).${ }^{15}$ Pinero et al have later supported this finding with another system, the Pentacam (Oculus). ${ }^{18}$ The authors have reported a significantly greater posterior astigmatism of $0.56 \pm 0.28 \mathrm{D}$ in subclinical keratoconus compared to 0.33 $\pm 0.24 \mathrm{D}$ in normal eyes. More recently, we also reported similar results with the Galilei system (Ziemer inc), with significant differences between normal corneas and forms fruste keratoconus in posterior elevation, posterior peripheral keratometry and posterior I-S value. ${ }^{19}$

However, no consensus is accepted concerning discriminant variables and cutoff values at the level of the posterior surface for differentiating normal corneas from early $\mathrm{KC}$ because of the high discrepancy in results between 
studies. The use of different technologies for imaging the cornea, as well as the definition of subclinical $\mathrm{KC}$, which vary a lot between studies, explain these discrepancies. Discriminant parameters and their cutoff values should, therefore, be properly used with the appropriate imaging system. Rao et al have set the posterior elevation cutoff with the Orbscan IIz system at $40 \mu \mathrm{m}$ in suspect topographies, for recusing patients for LASIK. ${ }^{20}$ Mihaltz et al reported a posterior elevation cutoff value with the Pentacam system of $15.5 \mu \mathrm{m}$ for discriminating normal eyes from keratoconus, with $95.1 \%$ sensitivity and $94.3 \%$ specificity. ${ }^{21}$ With the same technology but different setting, De Sanctis et al also found posterior corneal elevation very effective for differentiating keratoconus from normal corneas with a sensitivity and specificity of 97.3 and $96.9 \%$ respectively and a cutoff value of $38 \mu$ m over a $5 \mathrm{~mm}$ diameter BFS. ${ }^{22}$ However, the efficacy was lower for subclinical keratoconus with respectively 68 and $90.8 \%$ and a cut-off value of $32 \mu \mathrm{m}$. This finding has led the authors to preconize not using posterior elevation value alone for stratifying the keratoconus condition. Uçakhan et al have later suggested a composite index combining several posterior elevation derived parameters, such as the maximum posterior elevation, the maximum posterior depression and the difference between both as well as corneal power for improving the sensitivity of the screening test for differentiating normal cornea from subclinical keratoconus. ${ }^{23}$ The authors reported that this combination of posterior elevation data with corneal power yielded better predictive accuracy in keratoconus and subclinical keratoconus eyes. Nilforoushan et al performed a multiple regression analysis and have identified the larger difference between the highest and lowest points on the posterior elevation maps with both Pentacam and Orbsacn IIz system, as the strongest predictor of suspect keratoconus. ${ }^{24}$

More recently, studies using artificial intelligence systems for discriminating subclinical KC from normal corneas have further supported the leading current hypothesis that keratoconic disease may be first detectable at the posterior surface. ${ }^{11,25,26}$ Modifications of the posterior surface are of particular interest in the mildest form of the disease in the sense that corneal epithelium has been shown to have the potential of smoothing corneal topographic irregularities and masking the presence of an underlying cone on the anterior surface of mild keratoconus. ${ }^{14,27}$ Saad and Gatinel have developed a very sensitive screening test with the Orbscan IIz system, using a discriminant function generated from corneal thickness, curvature and elevation measurements and have suggested that posterior surface modifications concomitant to a paracentral corneal thinning may be the first sign of subclinical KC. ${ }^{11}$ More recently, our group developed an automated detection program for subclinical $\mathrm{KC}$ with the Galilei Analyzer system, using a machine- learning classifier. ${ }^{25}$ This artificial intelligence system has the ability to automatically select the most discriminant parameters with its respective cutoff values among all the variables analyzed, for differentiating your populations. Interestingly, parameters related to posterior surface asymmetry and corneal volume were chosen by the system as the most discriminant for identifying the subclinical KC. Using another imaging system, the Cirius (CSO inc) and another artificial intelligence method, Arbelaez et al have also suggested that measurements derived from the posterior surface significantly helped to improve the sensitivity of subclinical KC detection. ${ }^{26}$

\section{Elevation Parameters and Role of the Reference Body Selection}

Elevation data are represented relative to reference shapes so that the clinician does not analyze the actual elevation data but data after subtracting out the reference shape. This method has been used in order to magnify the differences and allow for qualitative maps that will highlight clinically significant areas. ${ }^{28}$ Very few studies in the literature have addressed the influence of the reference shape for diagnosing keratoconus. Kovacs et al have compared the discriminating ability and predictors of the posterior elevation values obtained by both, the best fit sphere (BFS) and the best fit toric ellipsoid (BFTE) reference surfaces. ${ }^{29}$ The BFTE incorporates the difference in curvature between the two principal meridians as well as the corneal asphericity. The authors reported that the use of the BFTE was associated with a decreased risk of masking keratoconus cases and had a significantly better ability for discriminating keratoconus from normal subjects than that with the BFS. The sensitivity and specificity were respectively 95 and $97 \%$ with the BFTE and 91 and $98 \%$ with the BFS. More recently, using the Galilei Analyzer, we demonstrated that the ability to discriminate between normal corneas and FFKC with elevation parameters was significantly improved by using an aspherotoric reference surface shape (BFTA) rather than the classical spherical shape (BFS). ${ }^{30}$ In addition, receiver operator characteristic curve (ROC) analysis showed that posterior elevation measured with the BFTA had a significantly higher predictive accuracy for identifying FFKC than anterior elevation with an area under ROC curves of 0.88 and 0.80 , respectively. The sensitivity and specificity achieved with the posterior elevation for detecting $\mathrm{KC}$ and FFKC were 99 and $99 \%$ for KC and 82 and $80 \%$ for FFKC by setting the cutoff value at 16 and $13 \mu \mathrm{m}$, respectively.

The rational behind this finding is that the aspherical and toric reference surface lies closer to the natural shape of the cornea. This way, it will minimize the influence of corneal toricity and asphericity on elevation calculation, which are responsible for the commonly seen 'ridge pattern' 
on elevation maps. ${ }^{30}$ Therefore, it might help revealing the fine abnormalities that deviate from a regular aspherotoric surface and would be otherwise hidden by the ridge pattern seen when calculated relative to a spherical surface. This difference between the BFS and BFTA displays becomes particularly relevant when tracking subtle abnormalities in elevation maps for detecting subclinical $\mathrm{KC}$.

\section{Indices based on Pachymetry}

Corneal thinning has been shown to be a key pathologic feature of keratoconus. ${ }^{24,31}$ However, Rabinowitz et al have pointed out the inability of ultrasonic pachymetry to accurately detect the location of corneal thinning in keratoconus by measuring standard points on the cornea. ${ }^{32}$ Therefore, the authors have suggested not relying on this method for distinguishing between keratoconus and normal cornea given the unacceptable high rate of false-negative with only $86 \%$ of keratoconic patients correctly classified as keratoconus.

Over the last decade, full thickness analysis has gained in clinical relevance with the introduction of corneal tomography allowing a three-dimensional (3-D) reconstruction of the cornea with the creation of a pachymetric map along with the identification of the thinnest pachymetric (TP) value and its location. In addition, corneal tomography can provide data for characterizing the corneal thickness distribution. Following the pioneering investigations of Mandell and Polse on corneal thickness profile, ${ }^{33}$ the works of Ambrosio et al with the Pentacam system have led to the development of novel pachymetry-based indices that helped to better discriminate normal eyes from keratoconus. ${ }^{34-36}$ Corneal thickness at the thinnest point and the averages of points along 22 imaginary circles centered on the thinnest point with increased diameters of $0.4 \mathrm{~mm}$ steps were calculated to create what the authors called the 'corneal-thickness spatial profile' (CTSP). They also introduced the concept of percentage increase in thickness (PIT) calculated for each position of the corneal-thickness spatial profile from their first value. In a first study, the authors showed that the CTSP and the PIT were significantly different between keratoconic corneas and normal corneas. ${ }^{34}$ They found that keratoconic eyes have thinner corneas than normal eyes, with less volume and a more abrupt increase in PIT and CTSP from the thinnest point toward the periphery. This finding was later supported by the work of Saad and Gatinel with Orbscan, who went even further by showing that PIT was already significantly higher in the most incipient form of the keratoconus disease (FFKC) than in normal corneas. ${ }^{11}$ Previous studies had already reported thinner corneas in suspect keratoconus than in normal eyes ${ }^{15,24}$ and a progressive thinning along with the progression of the disease. ${ }^{18}$ However, the finding of Saad et al suggests that subclinical KC might be characterized not only by thinner corneas but also by a quick modification of the corneal thickness from the thinnest point to the periphery.

More recently, Ambrosio et al have described the concept of relational thickness, which is the ratio between singlepoint metrics (TP and CCT) and values of the pachymetric progression indices (PPI) ${ }^{35}$ The authors found a sensitivity of $100 \%$ and a specificity of $95.6 \%$ for differentiating normal from keratoconic corneas with the Ambrosio relational thickness (ART) index, thereby having a better discriminating ability than single-point pachymetric measurements. The performance of this new index still remains to be tested and validated for the detection of early keratoconus.

Pachymetry-based indices have also been used for refining the grading of the keratoconus disease. Prakash and Agarwal have recently determined pachymetric cutoff values with the Orbscan IIz system, as adjuncts to the existing keratometric criteria to screen keratoconus suspects. ${ }^{37}$ Their findings suggested that a cornea that had a TP $<461 \mu \mathrm{m}$ or a difference between the TP and the central corneal thickness greater than $27 \mu \mathrm{m}$ has $97.5 \%$ of risk of being suspect or worse, and thus, only $2.5 \%$ chance of being normal.

\section{CONCLUSION}

Along with the latest advances in anterior segment imaging and the ability to analyze the posterior corneal surface as well as the pachymetric profile in addition to the anterior surface curvature, new corneal indices have emerged as key parameters for improving the sensitivity subclinical $\mathrm{KC}$ detection. However, the keratoconus disease, being a complex and still not fully understood eye condition, the analysis of corneal properties such as wavefront and biomechanics, in combination to morphological corneal characteristics (topographic and tomographic), will certainly further improve the sensitivity of the future screening tests.

\section{REFERENCES}

1. Maeda N, Klyce S, Tano Y. Detection and classification of mild irregular astigmatism in patients with good visual acuity. Surv Ophthalmol 1998;43(1):53-58.

2. Randleman JB, Russell B, Ward MA, Thompson KP, Stulting RD. Risk factors and prognosis for corneal ectasia after LASIK. Ophthalmology 2003;110(2):267-275.

3. Maeda N, Klyce SD, Smolek M. Automated keratoconus screening with corneal topography analysis. Invest Ophthalmol Vis Sci 1994;35:2749-2757.

4. Chastang PJ, Borderie VM, Carvajal-gonzalez S, Roste W. Automated keratoconus detection using the EyeSys videokeratoscope. J Cataract Refract Surg 2000;26:675-683.

5. Rabinowitz YS, McDonnell P. Computer-assisted corneal topography in keratoconus. Refract Corn Surg 1989;5:400-408.

6. Rabinowitz YS, Rasheed K. KISA\% index: a quantitative videokeratography algorithm embodying minimal topographic 
criteria for diagnosing keratoconus. J Cataract Refract Surg 1999;25:1327-1335.

7. Maeda N, Klyce SD, Smolek M. Comparison of methods for detecting keratoconus using videokeratography. Arch Ophthalmol 1995;113(7):870-874.

8. Abad JC, Rubinfeld RS, Del Valle M, Belin MW, Kurstin JM. Vertical D: a novel topographic pattern in some keratoconus suspects. Ophthalmology 2007;114(5):1020-1026.

9. Mahmoud AM, Roberts CJ, Lembach RG, et al. The cone location and magnitude index. Cornea 2008;27(4):480-487.

10. Belin MW, Ambrosio R. Corneal ectasia risk score: statistical validity and clinical relevance. J Refract Surg 2010;26(4):238-241.

11. Saad A, Gatinel D. Topographic and tomographic properties of forme fruste keratoconus corneas. Invest Ophthalmol Vis Sci 2010;51(11):5546-5555

12. Gatinel D, Racine L, Hoang-Xuan T. Contribution of the corneal epithelium to anterior corneal topography in patients having myopic photorefractive keratectomy. J Cataract Refract Surg 2007;33(11):1860-1865.

13. Reinstein DZ, Cantab MA, Gobbe M, et al. Epithelial, stromal, and total corneal thickness in keratoconus: three-dimensional display with artemis very-high frequency digital ultrasound. J Refract Surg 2010;26(4):259-272.

14. Touboul D, Trichet E, Binder PS, et al. Comparison of front-surface corneal topography and Bowman membrane specular topography in keratoconus. J Cataract Refract Surg 2012;38(6):1043-1049.

15. Schlegel Z, Hoang-Xuan T, Gatinel D. Comparison of and correlation between anterior and posterior corneal elevation maps in normal eyes and keratoconus-suspect eyes. J Refract Surg 2007:789-795.

16. Ambròsio RJ, Dawson DG, Salomao M, et al. Corneal ectasia after LASIK despite low preoperative risk: tomographic and biomechanical findings in the unoperated, stable, fellow eye. J Refract Surg 2010;26(11):906-911.

17. Tomidokoro A, Oshika T, Amano S, et al. Changes in anterior and posterior corneal curvatures in keratoconus. Ophthalmology. 2000;107(7):1328-1332.

18. Piñero DP, Alió JL, Alesón A, Escaf Vergara M, Miranda M. Corneal volume, pachymetry, and correlation of anterior and posterior corneal shape in subclinical and different stages of clinical keratoconus. J Cataract Refract Surg 2010;36(5):814-825.

19. Smadja D, Touboul D, Colin J. Comparative evaluation of elevation, keratometric, pachymetric and wavefront parameters in normal eyes, subclinical keratoconus and keratoconus with a dual scheimpflug Analyzer. Int J Kerat Ect Cor Dis 2012;1(10): $1-9$.

20. Rao SN, Raviv T, Majmudar PA, Epstein RJ. Role of Orbscan II in screening keratoconus suspects before refractive corneal surgery. Ophthalmology 2002;6420(02):1642-1646.

21. Miháltz K, Kovács I, Takács A, Nagy ZZ. Evaluation of keratometric, pachymetric, and elevation parameters of keratoconic corneas with pentacam. Cornea 2009;28(9):976-980.

22. de Sanctis U, Loiacono C, Richiardi L, et al. Sensitivity and specificity of posterior corneal elevation measured by Pentacam in discriminating keratoconus/subclinical keratoconus. Ophthalmology 2008;115(9):1534-1539.

23. Uçakhan ÖÖ, Cetinkor V, Özkan M, Kanpolat A. Evaluation of Scheimpflug imaging parameters in subclinical keratoconus, keratoconus, and normal eyes. J Cataract Refract Surg 2011;37(6):1116-1124.
24. Nilforoushan M-reza, Speaker M, Marmor M. Comparative evaluation of refractive surgery candidates with Placido topography, Orbscan II, Pentacam and wavefront analysis. J Cataract Refract Surg 2008;34:623-631.

25. Smadja D, Touboul D, Cohen A, et al. Detection of subclinical keratoconus using an automated decision tree classification. Am J Ophthalmol 2013;156(2):237-246.

26. Arbelaez MC, Versaci F, Vestri G, Barboni P, Savini G. Use of a support vector machine for keratoconus and subclinical keratoconus detection by topographic and tomographic data. Ophthalmology. 2012;119:2231-2238.

27. Reinstein DZ, Archer TJ, Gobbe M. Corneal epithelial thickness profile in the diagnosis of keratoconus. J Refract Surg 2009;25:604-610.

28. Belin MW, Khachikian SS. An introduction to understanding elevation-based topography : how elevation data are displayed - a review. Clin Experiment Ophthalmol 2008;(1):1101-1106.

29. Kovács I, Miháltz K, Ecsedy M, Németh J, Nagy ZZ. The role of reference body selection in calculating posterior corneal elevation and prediction of keratoconus using rotating Scheimpflug camera. Acta Ophthalmologica 2011;89(3):251-256.

30. Smadja D, Santhiago MR, Mello GR, et al. Influence of the reference surface shape for discriminating between normal corneas, Subclinical Keratoconus and Keratoconus. J Refract Surg 2013;29(4):274-281.

31. Gatinel D, Malet J, Hoang-Xuan T. Corneal elevation topography: best fit sphere, elevation distance, asphericity, toricity and clinical implications. Cornea 2011;30(5):508-515.

32. Lim L, Wei RH, Chan WK, Tan DTH. Evaluation of keratoconus in Asians: role of Orbscan II and Tomey TMS-2 corneal topography. Am J Ophthalmol 2007;143(3):390-400.

33. Rabinowitz YS. Accuracy of ultrasonic pachymetry and videokeratography in detecting keratoconus. J Cataract Refract Surg 1998;24(2):196-201.

34. Mandell R, Polse K. Keratoconus: spatial variation of corneal thickness as a diagnostic test. Arch Ophthalmol 1969;82:182-188.

35. Ambrósio R, Alonso RS, Luz A, Guillermo L, Velarde C. Corneal-thickness spatial profile and corneal-volume distribution: tomographic indices to detect keratoconus. J Cataract Refract Surg 2006;32(11):1851-1859.

36. Ambrósio R, Caiado ALC, Frederico P, Louzada R, Roy AS, Luz A, Dupps WJ, Belin MW. Novel pachymetric parameters based on corneal Tomography for diagnosing Keratoconus. J Refract Surg 2011;27(10):753-758.

37. Ambrósio R. Percentage thickness increase and absolute difference from thinnest to describe thickness profile. J Refract Surg 2010;26(2):84-86.

38. Prakash G, Agarwal A, Mazhari AI, et al. A new pachymetrybased approach for diagnostic cutoffs for normal, suspect and keratoconic cornea Eye 2012;26(5):650-657.

\section{ABOUT THE AUTHOR}

\section{David Smadja}

Refractive Surgeon, Department of Ophthalmology, Anterior Segment and Refractive Surgery Unit, Hopital Pellegrin, University Hospital of Bordeaux; National Reference Center for Keratoconus (CRNK) Bordeaux, France, Phone: 0033556795679, e-mail: davidsmadj@ hotmail.fr 